University of Wollongong

Research Online

Faculty of Engineering and Information

Faculty of Engineering and Information

Sciences - Papers: Part B

Sciences

2017

Statistical Shape Model Generation Using Diffeomorphic Surface

Registration

Jiaqi Wu

Tianjin Polytechnic University

Guangxu Li

Tianjin Polytechnic University

Huimin Lu

Kyushu Institute of Technology

Hyoung Kim

Kyushu Institute of Technology

Philip O. Ogunbona

University of Wollongong, philipo@uow.edu.au

Follow this and additional works at: https://ro.uow.edu.au/eispapers1

Part of the Engineering Commons, and the Science and Technology Studies Commons

Research Online is the open access institutional repository for the University of Wollongong. For further information contact the UOW Library: research-pubs@uow.edu.au 


\title{
Statistical Shape Model Generation Using Diffeomorphic Surface Registration
}

\begin{abstract}
Statistical shape modelling is an efficient and robust method for medical image segmentation in computer-aided diagnosis. The key step in building a statistical shape model is to find corresponding landmarks in each instance of a training set. In this paper, a novel landmark correspondence estimation method that uses edge collapse surface simplification and the sphere registration is proposed. All the landmarks are selected and transformed by spherical conformal mapping from the instances of the training set and the associated correspondence are automatically found on the spheres. We applied our method on 21 cases of 3-D right lung shapes. The results of image segmentation experiment indicate that our method has a positive influence on the accuracy of segmentation result.
\end{abstract}

\section{Keywords}

surface, model, registration, diffeomorphic, statistical, generation, shape

Disciplines

Engineering | Science and Technology Studies

\section{Publication Details}

Wu, J., Li, G., Lu, H., Kim, H. \& Ogunbona, P. O. (2017). Statistical Shape Model Generation Using Diffeomorphic Surface Registration. ICBIP 2017: Proceedings of the 2nd International Conference on Biomedical Signal and Image Processing (pp. 37-41). New York, United States: ACM. 


\section{Statistical Shape Model Generation Using Diffeomorphic Surface Registration}

\author{
J. Wu \\ School of Electronics and \\ Information Engineering, \\ Tianjin Polytechnic University, \\ No. 399, Binshui Xi Road, \\ Xiqing District, Tianjin, \\ 300387, China \\ wujiaqi_alan@outlook.com
}

G. Li

School of Electronics and Information Engineering,

Tianjin Polytechnic University,

No. 399, Binshui Xi Road, Xiqing District, Tianjin, 300387, China

liguangxu@tjpu.edu.cn

\author{
H. Lu \\ Department of Control Engineering, \\ Kyushu Institute of Technology, \\ 1-1 Sensuicho, Tobata, Kitakyushu, \\ Fukuoka, Japan \\ lu@cntl.kyutech.ac.jp
}

\author{
P. O. Ogunbona \\ School of Computing and Information Technology, \\ University of Wollongong, \\ Northfield Avenue, Wollongong, NSW 2522, Australia \\ philipo@uow.edu.au
}

\begin{abstract}
Statistical shape modelling is an efficient and robust method for medical image segmentation in computer-aided diagnosis. The key step in building a statistical shape model is to find corresponding landmarks in each instance of a training set. In this paper, a novel landmark correspondence estimation method that uses edge collapse surface simplification and the sphere registration is proposed. All the landmarks are selected and transformed by spherical conformal mapping from the instances of the training set and the associated correspondence are automatically found on the spheres. We applied our method on 21 cases of 3-D right lung shapes. The results of image segmentation experiment indicate that our method has a positive influence on the accuracy of segmentation result.
\end{abstract}

\section{CCS Concepts}

- Computing methodologies $\rightarrow$ Artificial intelligence $\rightarrow$ Computer vision $\rightarrow$ Computer vision problem $\rightarrow$ Image segmentation.

\section{Keywords}

Statistical shape model; correspondence; surface simplification; surface registration.

\section{INTRODUCTION}

Statistical shape models (SSM) are widely used in the medical image segmentation on account of their high accuracy and robustness to noise. Generally, the model is represented by the point distribution model (PDM), which contains a set of labelled

Permission to make digital or hard copies of all or part of this work for personal or classroom use is granted without fee provided that copies are not made or distributed for profit or commercial advantage and that copies bear this notice and the full citation on the first page. Copyrights for components of this work owned by others than ACM must be honored. Abstracting with credit is permitted. To copy otherwise, or republish, to post on servers or to redistribute to lists, requires prior specific permission and/or a fee. Request permissions from Permissions@acm.org

ICBIP '17, August 23-25, 2017, Kitakyushu, Japan

(C) 2017 Association for Computing Machinery.

ACM ISBN 978-1-4503-5268-0/17/08 ..\$15.00

https://doi.org/10.1145/3133793.3133796 points, called landmarks, extracted from the volume data [1]. The key step of constructing a robust and effective point model is to find the correspondence of each instances, which directly determines the quality of the model and the result of segmentation. Methods that depend on manual estimation of landmark correspondence on 3-D surfaces are time consuming and errorprone. Automatic methods on the other hand suffer the problem of effective quantitative definition of correspondence.

Davies et al. [2] described the quantitative evaluation criteria for global description of SSMs. They proposed an objective function method based on the minimum description length (MDL) and pose the model construction as an optimization problem. The complexity and the fitting quality of the model are balanced by the MDL in a two-part coding formulation. Heimann et al. [3] employed a gradient descent optimization method to minimize the description length cost function and achieved easier and faster implementation than those of [2]. The positions of the landmarks are modified locally without disturbing established correspondence. Several orders of magnitude are used to accelerate the model building and a singular value decomposition is employed to estimate a more accurate gradient information.

We focus on the local surface features correspondence methods, which take advantage of image segmentation. In our previous modelling method [4], the landmarks are generated from a reference sphere whose landmarks are equally distributed. After a spherical conformal mapping, the landmarks are directly found on each mapped sphere while neglecting the different poses of shapes extracted from the images and the deformation of the vertices position. This paper tackles the drawbacks of the previous method and proposes a correspondence method based on sphere registration to automatically find the landmarks on each training instance. The registration method is performed on the spheres before estimating the correspondence between the reference sphere and the spheres of other instances.

In the following section, we present an improved landmark distribution method. Here, a quadric-based simplification method is used to automatically generate landmarks in the reference shape. The registration of the sphere mapped by SPHARM is used to find corresponding vertices in each surface of the instances. In section three, the method is tested on right lung triangular meshes 
and a segmentation experiment is performed with the constructed model. An evaluation of the effectiveness of the model as well as the discussion of the segmentation result are introduced in the last section.

\section{METHOD}

The flowchart of our method is shown in Fig.1. First, one instance of surface is selected from the training set and a surface simplification method is performed on the instance, which generates a simplified surface. The vertices on the simplified surface are regarded as the reference landmarks. Then, the instance is transformed to the spherical parametric domain using spherical conformal mapping method. At the same time, the reference sphere is generated from the landmarks mapped to the sphere. Next, all the instances of the training set are mapped to the spheres in a similar manner. The gradient of each point in the instances of the training set is calculated and spherical registration is performed on the spheres generated previously by referring to the gradients of their corresponding points. After the registration, the correspondence landmarks are found on the other spheres by the reference sphere and tracked back on the surfaces of the instances.

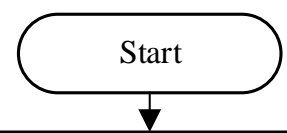

Select an instance surface from the training se
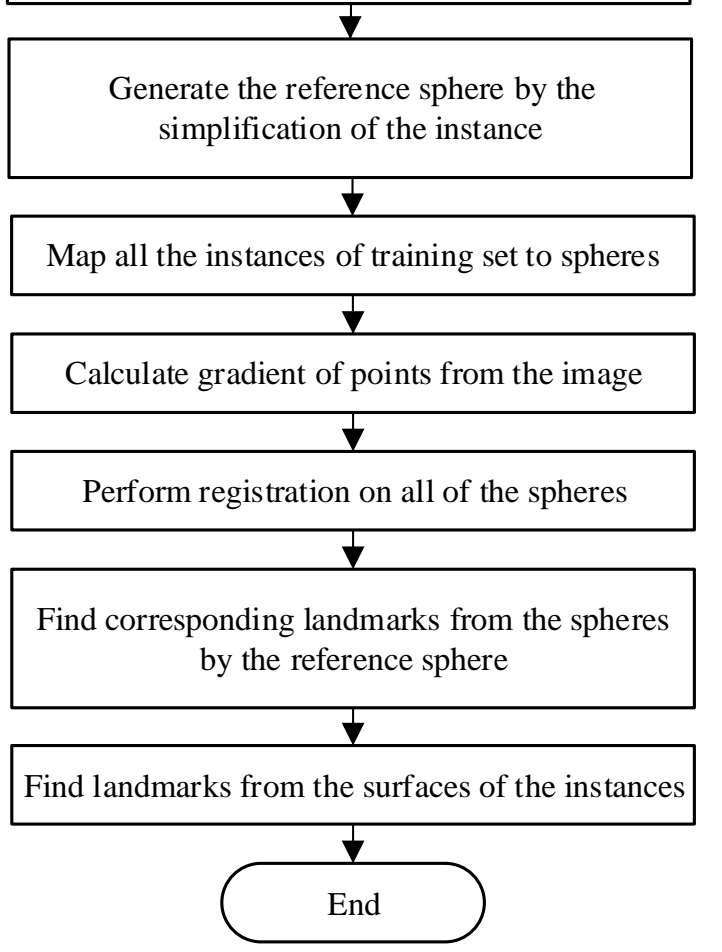

Figure 1. Flow chart of our proposed method.

The conformal mapping algorithm in our method is based on those described in [5]. The algorithm maps 0-genus surfaces of the instances in the Cartesian system to unit sphere in the spherical coordinate system while retaining their topological structures. Fig. 2 shows the reference spheres. The left and right reference spheres in the figure are generated by the previous and our proposed methods, respectively.
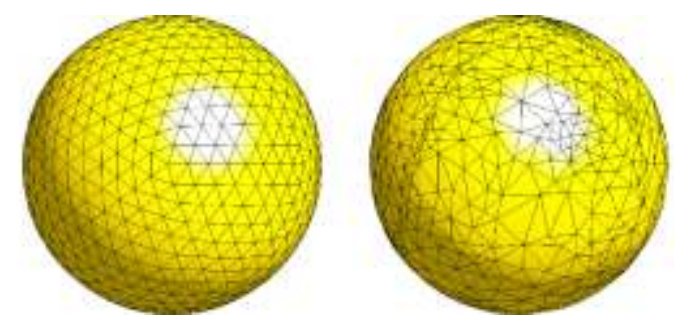

Figure 2. Reference spheres generated by the previous $\operatorname{method}($ left) and our proposed method (right).

We claim that the generation of the reference sphere is improved in this paper. In our previous method [4], the reference sphere is generated by a uniform triangular mesh in which the length of edges are equal. This implies that the landmarks are sampled by equal spaces from the surface. As a result, the landmarks in the region of higher curvature are coarser and those in the region of lower curvature are densely distributed. To obtain more representative landmarks in different curvatures of the surface, an efficient surface simplification algorithm using the quadric error metric is used in our proposed method. This algorithm uses iterative contractions of vertex pairs to simplify models and maintains surface error approximations using quadric matrices [6]. Fig. 3 shows the meshes of landmarks generated by the previous method and our proposed method. Careful inspection of Fig. 3 shows that more faces are used to express shapes with higher curvature in our proposed method.
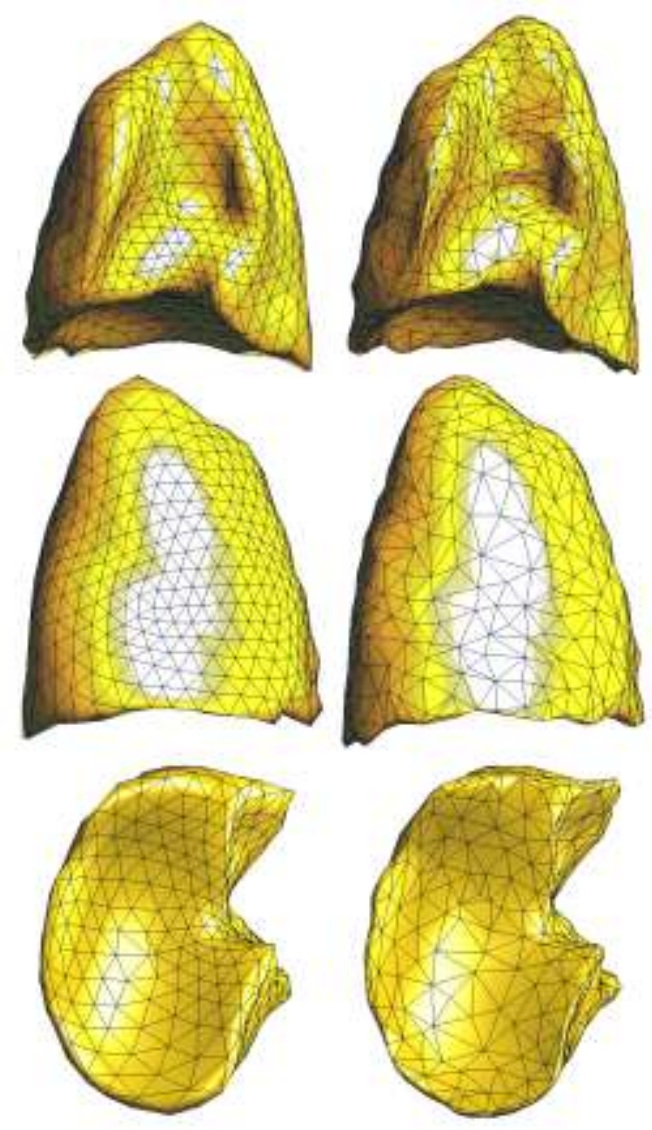

Figure 3. Meshes of the landmarks generated by the previous method (left column) and our proposed method (right column) from the same instance in their front, back and bottom views. 
As previously described, the positions of the vertices on the instances in spherical domain vary a lot in the spherical mapping process. To find the corresponding landmarks on the spheres, we introduce a sphere registration algorithm which uses a limited class of diffeomorphisms combined with the Demons algorithm [7]. The gradient value of each point is calculated from the original image as the reference for the registration. Because the indices of the vertices remain the same, the corresponding landmarks of each sphere can be labelled from the spheres under the registration by tracking the indices of their vertices which are nearest to the landmarks on the reference sphere. Note that the landmarks of the instances can be found by the indices as well.

\section{EXPERIMENT}

\subsection{Data Preparation}

For our study, 21 cases of thoracic computed tomography (CT) from the LIDC (Lung Image Database Consortium) are used. The size of the images varies from $144 \times 144 \times 127$ to $180 \times 180 \times$ 205 voxels and the gap between the voxels is $2 \mathrm{~mm}$. The training set is obtained by the method described in [4]. First, the CT images are pre-processed to enhance the lung region. Second, the region of interest (ROI) is extracted from the CT images by using the region growing method and represented by the binary voxel data. Third, the Marching Cubes algorithm is employed to generate a surface mesh while maintaining the inter-slice connectivity. Finally, a smoothening mean filter is applied to each surface to remove its roughness. In out experiment, the surfaces consist of 69676 vertices and 139349 faces on average. After the correspondence, each mesh obtained by the classic method and our method both contain 642 vertices and 1280 faces.

\subsection{Evaluation of Model Quality}

An objective method of evaluating model quality is introduced by [7]. Three properties are described as the indicators of model qualities: generalization ability, specificity and compactness.

The generalization ability of a model is used to identify the ability to construct an instance of shape that is not in the limited training set. It is usually implemented by the leave-one-out protocol. If for two methods A and B with $G_{A}$ and $G_{B}$ denoting the corresponding generalization ability and $M$ the number of shapes used to express the current instance, $G_{A}(M) \leq G_{B}(M)$ is for all of the $M$ and $G_{A}(M)<G_{B}(M)$ is for some of the $M$, we consider method $\mathrm{A}$ is better than method $\mathrm{B}$ in the generalization ability.

Specificity describes the similarity of the shape constructed and the shapes of the training set. Each shape in the training set is compared with the shapes generated by the model using a certain number of different parameters $b$. It can be described by:

$$
S(M)=\frac{1}{N} \sum_{j=1}^{N}\left|y_{j}(M)-y_{j}{ }^{\prime}\right|^{2}
$$

where $y_{j}$ is the shape constructed by the model and $y_{j}{ }^{\prime}$ is the most similar shape in the training set. Similar to the generalization ability, with $S_{A}(M) \leq S_{B}(M)$ for all of the $M$ and $S_{A}(M)<$ $S_{B}(M)$ for some of the $M$, we consider method A is more specific.

Compactness is the ability of constructing the instance with less parameters by the model. Supposing the $\lambda_{i}$ is the $i$ th eigenvalue, the compactness is described by the cumulative variance:

$$
C(N)=\sum_{i=1}^{N} \lambda_{i}
$$

For $C_{A}(M)$ and $C_{B}(M)$ which represent the compactness of method $\mathrm{A}$ and $\mathrm{B}$, comparison is similar to that of specificity.

\subsection{Evaluation of Segmentation Accuracy}

In our experiment, a leave-one-out protocol is used in the model construction and segmentation. The SSM contains shape model and appearance model. The shape model entails the statistics of the variation of landmark positions. An alignment is firstly performed by the Generalized Procrustes Analysis algorithm [8]. The scaling, translation and rotation among the training shapes are removed, which helps find the actual variation of the shapes. Then, the principal component analysis (PCA) is performed and the eigenvalues and their corresponding eigenvectors are calculated and used to construct the shape model. The appearance model is constructed by using similar procedure as the shape model. The intensity profiles of each landmark along the norm are sampled and used to automatically update the position of landmarks in the model searching process.

The segmentation using SSM can be regarded as a matching between the transformed model and the target structure of ROI in the image. This process can be summarized into two steps [9]:

In the first step, a shape instance of the model can be expressed by transformation $T$ and parameter $b_{s}$. The Mahalanobis distance defined in the appearance model is calculated along the norm vector of each landmark. In each norm, the point with the lowest Mahalanobis distance among the candidate points is selected as the new position of the landmark. The displacement vector of the positions of the landmarks can be expressed as $d y_{p}$.

In the second step, the generalized match is performed on $y$ and $y+d y_{p}$ to find a new transformation $T$ and new residual displacement $d y_{s}$.

These two steps are applied iteratively until a convergence criterion is met, e.g. the variation of movement is under a threshold or the iteration is up to a maximum.

The Dice similarity coefficient (DSC) method is used to evaluate the segmentation result with their volumetric overlap [10]. The overlap metric between two binary masks is defined as:

$$
D S C=\frac{2|A \cap B|}{|A|+|B|}
$$

where $A$ represents the experiment segmentation result and $B$ represents the gold standard from the expert. The DSC ranges between $[0,1]$ and $D S C=1$ denotes that the segmentation result is completely equal to the real shape.

\subsection{Results and Discussion}

Model quality evaluation and segmentation accuracy are obtained through experimentation and results depicted in Figs. 4 to Fig 7. The triangle symbol represents the model constructed by the previous method while the square symbol represents that of our proposed method.

The generalization ability, specificity and compactness of the models is compared and shown in Fig. 4 to Fig. 6 and the result of segmentation is shown in Fig. 7. The result shows that the proposed method is almost the same with the previous method in the model quality. The correspondence with local surface features does not seem to improve the model in the generalization ability, compactness and specificity which are the global characteristics of evaluating the model. However, our method results in a higher segmentation accuracy than the previous method, which implies that our method improves the SSM and provides an improved tool practical application. 


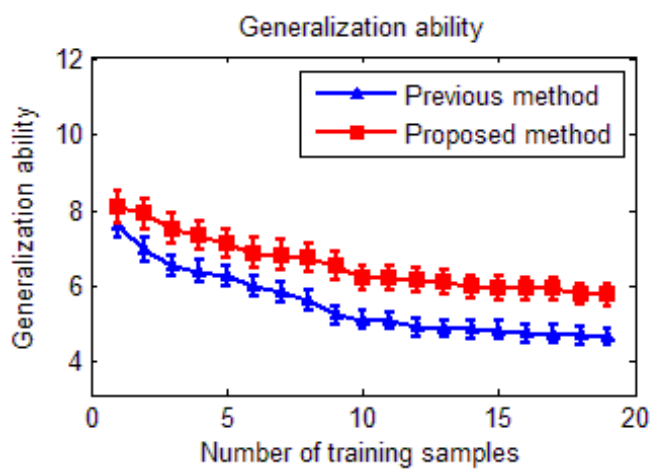

Figure 4. Generalization ability of the models constructed by the previous method and our proposed method.

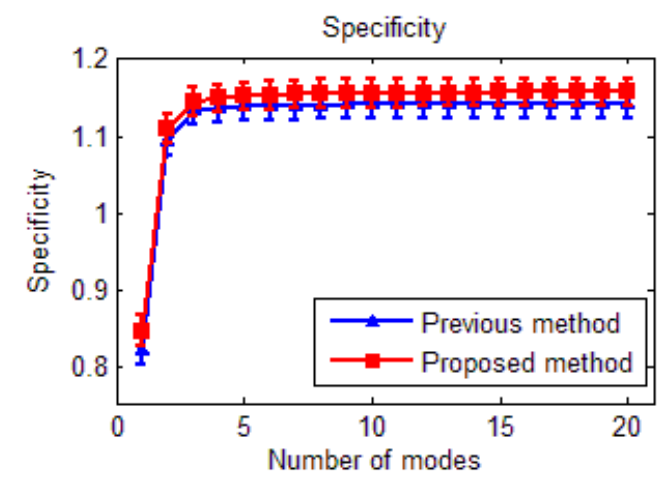

Figure 5. Specificity of the models constructed by the previous method and our proposed method.

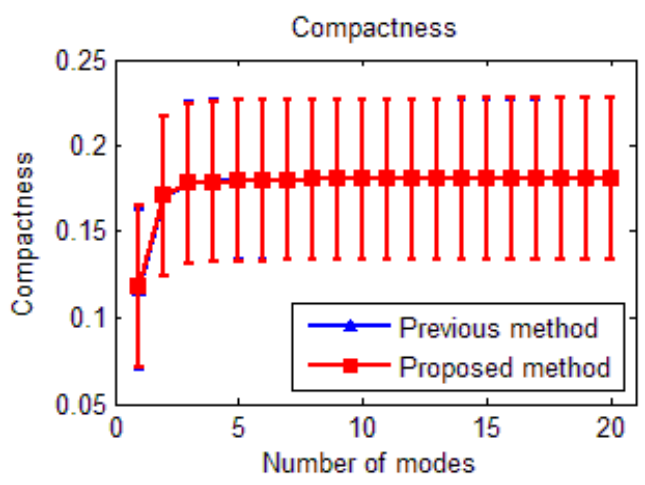

Figure 6. Compactness of the models constructed by the previous method and our proposed method.

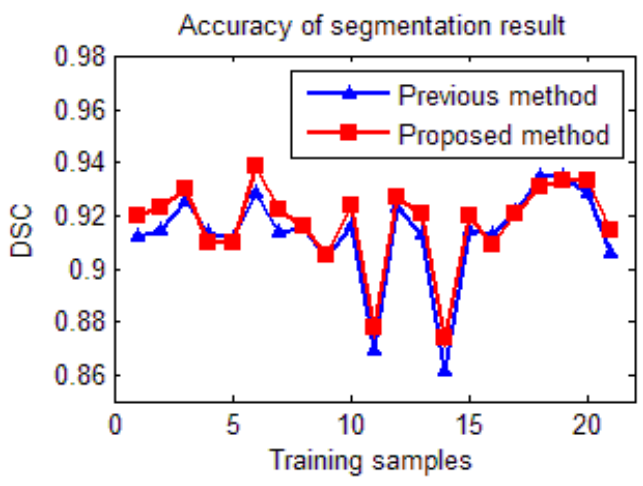

Figure 7. Accuracy of segmentation of the models constructed by the previous method and our proposed method.

\section{CONCLUSION}

In this paper, we presented a landmark correspondence method for the construction of SSM. A surface simplification method is used to generate landmarks of reference sphere. By referencing the gradient information in the registration process, a compact model is constructed. The experiment result shows that the model constructed by the proposed method and the previous method are almost the same in their generalization ability, compactness and specificity. However, the segmentation accuracy is improved by the proposed method. Future work will consider the use of more instances to train the model and improve its quality.

\section{ACKNOWLEDGMENTS}

All the images and gold standard data for research experiments were kindly provided by Seiichi Murakami at Hospital of the University of Occupational and Environmental Health, Japan.

Support for this research is provided by the Tianjin Application Foundation and Frontier Technology Research Project (14JCYBJC42300).

\section{REFERENCES}

[1] Cootes, T. F., Hill, A., Taylor, C. J., and Haslam, J. L. 1994. Use of active shape models for locating structures in medical images. Image Vis. Comput. 12, 6 (Jul.-Aug. 1994). 355-365. DOI=http://dx.doi.org/10.1016/0262-8856(94)90060-4.

[2] Davies, R. H., Twining, C. J., Cootes, T. F., Waterton, J. C., and Taylor, C. J. 2002. 3D statistical shape models using direct optimisation of description length. In European conference on computer vision (Copenhagen, Denmark, May 28-31, 2002). ECCV 2002. Springer, Berlin, Heidelberg, 320. DOI= http://dx.doi.org/10.1007/3-540-47977-5_1.

[3] Heimann, T., Wolf, I., Williams, T. G., and Meinzer, H.2005. $3 \mathrm{D}$ active shape models using gradient descent optimization of description length. In Biennial International Conference on Information Processing in Medical Imaging. (Glenwood Springs, CO, USA, July 10-15, 2005). IPMI 2005. Springer, Berlin, Heidelberg, 566-577. DOI= https://dx.doi.org/10.1007/11505730_47.

[4] Li, G. 2013. Study on the Method of Constructing a Statistical Shape Model and Its Application to the Segmentation of Internal Organs in Medical Images. Doctoral Thesis. Kyushu Institute of Technology.

[5] Gu, X., Wang, Y., Chan, T. F., Thompson, P. M., and Yau, S. 2004. Genus zero surface conformal mapping and its application to brain surface mapping. IEEE Trans. on Med. Im. 23, 8 (Aug. 2004), 949-958. DOI= https://dx.doi.org/10.1109/TMI.2004.831226.

[6] Garland, M., Heckbert, P. S. Surface simplification using quadric error metrics. 1997. In Proceedings of the 24th annual conference on Computer graphics and interactive techniques (Los Angeles, California, USA, August 03 - 08, 1997). SIGGRAPH '97. ACM Press/Addison-Wesley Publishing Co., New York, NY, USA, 209-216. DOI=http://dx.doi.org/10.1145/258734.258849.

[7] Yeo, B. T. T., Sabuncu, M., Vercauteren, T., Ayache, N., Fischl, B., and Golland, P. 2008. Spherical demons: Fast surface registration. In International Conference on Medical Image Computing and Computer-Assisted Intervention. (New York, NY, USA, September 6-10, 2008). MICCAI 2008. Springer Berlin Heidelberg, 745-753. DOI= https://dx.doi.org/10.1007/978-3-540-85988-8_89. 
[8] Gower, J. C. 1975. Generalized procrustes analysis. Psychometrika. 40, 1 (Mar. 1975), 33-51. DOI= https://dx.doi.org/10.1007/BF02291478.

[9] Heimann, T., Meinzer, H. P. 2009. Statistical shape models for 3D medical image segmentation: a review. Med. Image Anal. 13, 4 (Aug. 2009). 543-563. DOI= http://dx.doi.org/10.1016/j.media.2009.05.004.

[10] Sørensen, T. 1948. A method of establishing groups of equal amplitude in plant sociology based on similarity of species and its application to analyses of the vegetation on Danish commons. Biol. Skr. 5, 4 (Jan.1948). 1-34. 